\title{
Temperature Sensor Based on Ge-Doped Microstructured Fibers
}

\author{
Salvador Torres-Peiró, Antonio Díez, José Luis Cruz, and Miguel Vicente Andrés
}

Departamento de Física Aplicada-ICMUV, Universidad de Valencia, Dr. Moliner 50, 46100 Burjassot, Spain

Correspondence should be addressed to Miguel Vicente Andrés, miguel.andres@uv.es

Received 1 February 2009; Revised 14 May 2009; Accepted 8 July 2009

Recommended by Valerio Pruneri

The fundamental mode cutoff properties of Ge-doped microstructured fibers, filled with a liquid, permit the implementation of wavelength- and amplitude-encoded temperature sensors with an ultra-high sensitivity. The cutoff wavelength changes with temperature, and the thermo-optic coefficient of the liquid determines the sensitivity of the sensor. Sensitivity as high as $25 \mathrm{~nm} /{ }^{\circ} \mathrm{C}$ is reported. In addition, simple amplitude interrogation techniques can be implemented using the same sensor heads.

Copyright ( 2009 Salvador Torres-Peiró et al. This is an open access article distributed under the Creative Commons Attribution License, which permits unrestricted use, distribution, and reproduction in any medium, provided the original work is properly cited.

\section{Introduction}

A number of different approaches have been investigated along the last decades for the measurement of temperature using fiber-optics: Optical-scattering, fluorescence, interferometry, optical absorption, and so forth [1,2]. In-fiber Bragg gratings (FBGs) have been extensively investigated because they present several potential advantages: the robustness of wavelength codification, easy multiplexing, small size, and low cost [3]. The typical temperature sensitivity of FBG is $13 \mathrm{pm} /{ }^{\circ} \mathrm{C}$ at $1550 \mathrm{~nm}$, and it can be increased significantly by coating the FBG with other materials. A sensitivity of $108 \mathrm{pm} /{ }^{\circ} \mathrm{C}$ has been reported by coating the FBG with a polymer [4]. Using long period gratings (LPGs) larger sensitivities can be obtained $\left(310 \mathrm{pm} /{ }^{\circ} \mathrm{C}\right.$ was reported in [5]).

Other wavelength-encoded temperature sensors, that exhibit larger sensitivities than standard FBG, have been reported. The resonant coupling between the core-mode and the cladding modes in a special fiber with a multicladding structure has been used to make a temperature sensor, reporting a sensitivity of $240 \mathrm{pm} /{ }^{\circ} \mathrm{C}$ [6]. The temperature dependence of coupling coefficients in an optical fiber coupler with a special coating permitted to achieve $170 \mathrm{pm} /{ }^{\circ} \mathrm{C}$ sensitivity [7]. Finally, higher sensitivities, as high as $3.2 \mathrm{~nm} /{ }^{\circ} \mathrm{C}$, have been reported using short multimode fiber interferometers $[8,9]$.
Microstructured optical fibers open new opportunities to develop fiber sensors. Theoretical analysis of photonic crystal fiber sensors based on FBG and LPG predicts temperature sensitivities up to $60 \mathrm{pm} /{ }^{\circ} \mathrm{C}[10]$. Specially designed FabryPerot cavities formed with photonic crystal fibers have demonstrated sensitivities as high as $170 \mathrm{pm} /{ }^{\circ} \mathrm{C}$ [11].

Here we develop some details on the exploitation of a new concept, recently presented, of wavelength-encoded temperature sensor based on the temperature dependence of the cutoff wavelength of the fundamental mode in a liquid-filled Ge-doped microstructured fiber [12]. Photonic crystal fibers with a Ge-doped core have some appealing properties: (i) low-loss splicing to standard fibers, (ii) easy photoinscription of fiber gratings using conventional techniques, and (iii) good guidance when the holes are collapsed to build in-fiber gas o liquid cells. In addition, cutoff of the fundamental mode is produced when the holes are filled with liquids, as if it were a conventional depressedindex fiber [13]. Modifications of the characteristics of the liquids lead to changes of the guidance properties of the fiber, in particular, the cutoff wavelength shifts.

In our experiments, a sensitivity as high as $25 \mathrm{~nm} /{ }^{\circ} \mathrm{C}$ is achieved, which is about three orders of magnitude higher than the sensitivity of standard FBG, two orders of magnitude higher than the sensitivity of the best fiber gratings, and about one order of magnitude higher the values reported for multimode fiber interferometers. In addition 


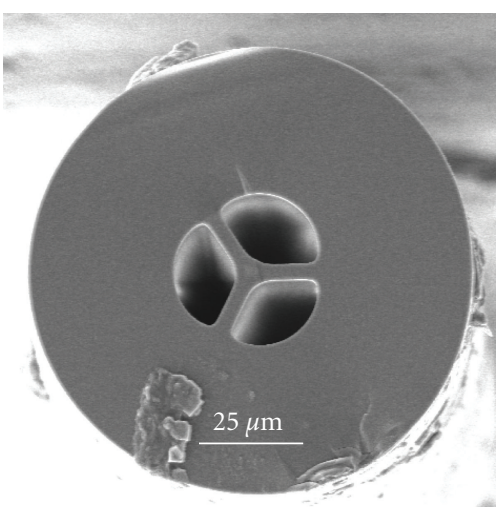

(a)

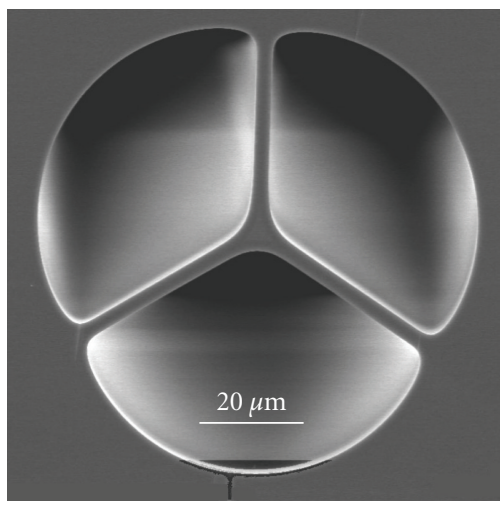

(c)

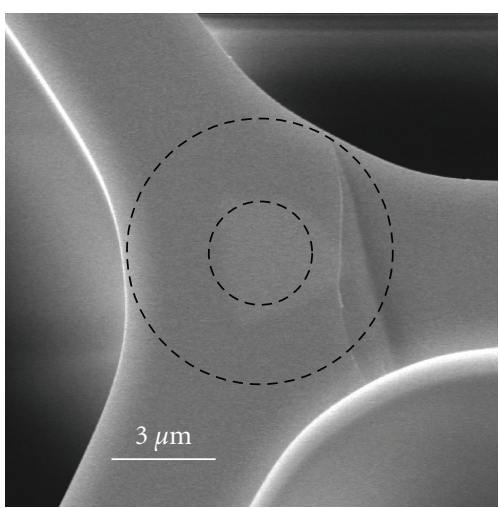

(b)

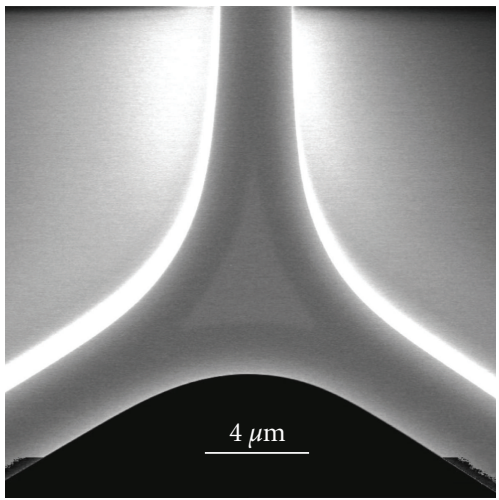

(d)

Figure 1: SEM images of fiber 1 (a) and (b) and fiber 2 (c) and (d).

to the wavelength-encoded configuration of the temperature sensor, we report here the amplitude interrogation of these devices. In this case, very simple experimental arrangements are required, preserving a high resolution.

\section{Principle}

The fiber that was used in our experiments is a Y-shaped Gedoped microstructured fiber. This fiber has three big holes, which makes straightforward the procedure to fill the fiber with a liquid. In addition, the Y-shaped fiber can be designed to have rather small core, hence, a strong interaction with the liquids filling the fiber due to the presence of intense evanescent fields in the holes. The fiber was fabricated in our laboratory with a Ge-doped core using the conventional stack and draw technique [12]. Figure 1 shows the scanning electron microscope (SEM) images of the two fibers reported here, with a detail of the core region. In the case of fiber 1 , two circles have been drawn on the image. The small circle is adjusted to have the same area that the triangular shaped Ge-doped region at the centre of the fiber, while the second circle is tangent to the three holes and defines what we call the first effective cladding of the fiber. The doped core had a Ge concentration to raise a step-index profile with a numerical aperture (NA) of 0.29 . The first cladding surrounding the core was pure silica. Table 1 summarizes the
TABLE 1: Basic parameters of the fibers.

\begin{tabular}{lccccc}
\hline & $d(\mu \mathrm{m})$ & $D(\mu \mathrm{m})$ & $O D(\mu \mathrm{m})$ & $A_{H}\left(\mu \mathrm{m}^{2}\right)$ & $d_{B}(\mu \mathrm{m})$ \\
\hline Fiber 1 & 3.3 & 8 & 110 & 1000 & 4.4 \\
Fiber 2 & 3.3 & 6 & 136 & 3570 & 2 \\
\hline
\end{tabular}

basic parameters of the two fibers used in our experiments: the diameter of the circle that corresponds to the Ge-doped core $(d)$, the diameter of the first effective cladding $(D)$, the outer diameter of the fiber $(O D)$, the holes' area $\left(A_{H}\right)$, and the silica bridges thickness $\left(d_{B}\right)$. The two fibers were singlemode when filled with liquids with a refractive index around 1.44 .

The liquids that we used for these experiments were provided by Cargille (series A) and have a nominal refractive index (RI) value of $1.46,1.47$, and 1.48 , measured at $589 \mathrm{~nm}$ and $25^{\circ} \mathrm{C}$. The temperature coefficient of these liquids, provided by the manufacturer, is about $-4 \times 10^{-4}{ }^{\circ} \mathrm{C}^{-1}$. Thus, when one fiber is filled with one of these liquids, the cutoff of the fundamental mode takes place at a given wavelength, as a function of the refractive index value. Figure 2 shows the typical transmission spectra for four devices with different lengths: 2, 18, 22, and $35 \mathrm{~cm}$. They have been measured with a broadband unpolarized light source and an optical spectrum analyzer (OSA). The devices of 2 and $22 \mathrm{~cm}$ length 


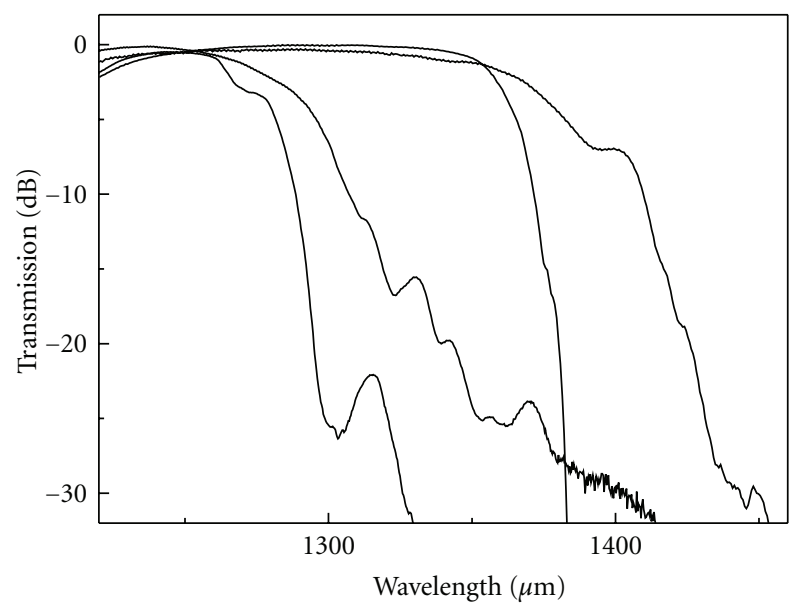

Figure 2: Transmission spectra of four sensor heads with different lengths, from left to right: $22,2,35$, and $18 \mathrm{~cm}$.

were prepared with fiber 2 (see Table 1) and filled with a liquid of RI $=1.475$ (a mixture of two liquids: $50 \%$ of 1.47 and $50 \%$ of 1.48 ), while the devices of 18 and $35 \mathrm{~cm}$ length were filled with $\mathrm{RI}=1.47$, and they were prepared with fiber 2 and fiber 1, respectively. After filling the holes, both ends of the fiber were spliced to standard singlemode fiber. We can see that the losses increase dramatically beyond the cutoff wavelength, even in the case of the short sensor. Though most of the results that we will present here have been obtained with devices that were about $20 \mathrm{~cm}$ long, this figure demonstrates that sensor heads ten times shorter can be used if it is required. At present, the optimization of the short sensor heads has not been concluded yet.

The operation principle of the temperature sensor was discussed in a previous publication [12]. The cutoff is produced when the dispersion curve of the fundamental mode intersects the dispersion curve of the liquid, that has a refractive index between the refractive index of silica and the refractive index of the Ge-doped silica. When the temperature changes, the thermo-optic coefficient of the liquid dominates the properties of the device, since silica has a coefficient 40 times smaller. A small change of the refractive index of the liquid shifts the point of intersection and produces a large change of the cutoff wavelength. The calculations of the cutoff wavelength as a function of temperature, using the step-index model, predict a linear response within the temperature range of our experiments, $[-10,60]^{\circ} \mathrm{C}$.

\section{Experimental Results and Discussion}

In order to illustrate the basic characteristics of these devices as temperature sensors, Figure 3(a) shows the transmission spectra of a sensor head that was prepared by filling $18 \mathrm{~cm}$ of fiber 2 (see Table 1) with liquid of RI 1.47. In this experiment the temperature was adjusted from $15^{\circ} \mathrm{C}$ to

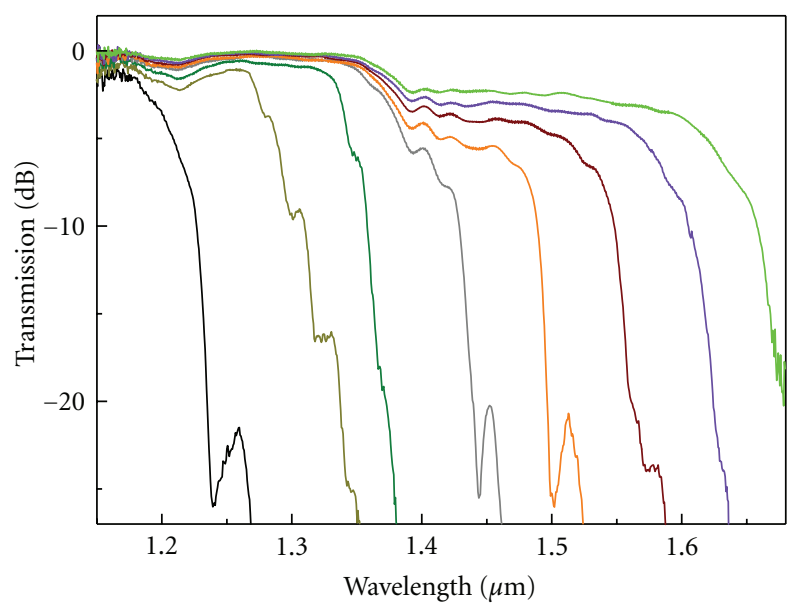

(a)

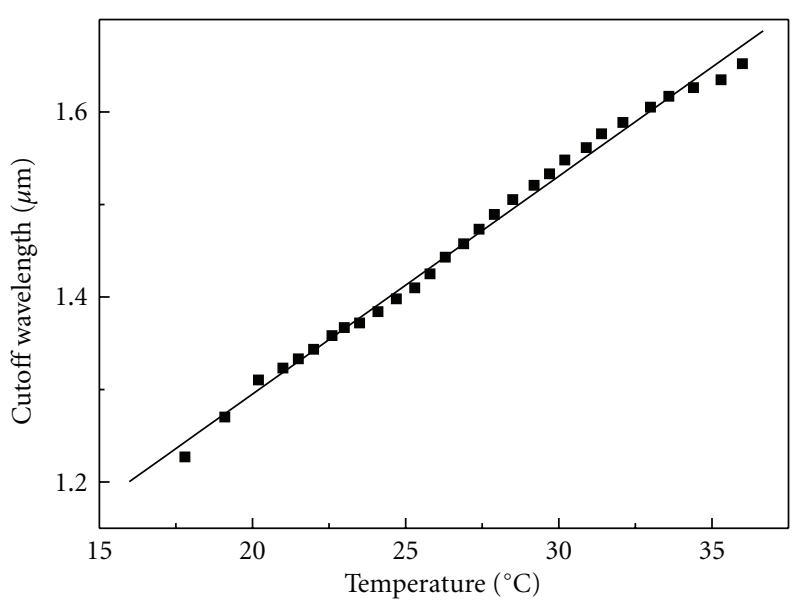

(b)

FIgURE 3: (a) Transmission spectra of a sensor head, with $18 \mathrm{~cm}$ length, at different temperatures, from left to right: 17.8, 20.2, 22.6, $25.8,27.9,30.2,33.0$, and $36.8^{\circ} \mathrm{C}$. (b) Calibration of this sensor head: cutoff wavelength as a function of temperature.

$35^{\circ} \mathrm{C}$ and the cutoff wavelength shifted from $1.2 \mu \mathrm{m}$ to $1.65 \mu \mathrm{m}$. This sensor head is the shortest that we have fully calibrated. Figure 3(b) gives the result of the calibration of the temperature response of this sensor head, taking the cutoff wavelength at the point the transmission drops $10 \mathrm{~dB}$. The sensitivity is $\sim 25 \mathrm{~nm} /{ }^{\circ} \mathrm{C}$, the same that was reported in [12] for longer devices.

Since the sensitivity is very high, one can preserve a good detection limit while using low resolution OSA. According to the $25 \mathrm{~nm} /{ }^{\circ} \mathrm{C}$ slope, a low-cost OSA with a resolution of $1 \mathrm{~nm}$ would permit to achieve a detection limit of $0.04^{\circ} \mathrm{C}$, while the use of an OSA with $20 \mathrm{pm}$ resolution would push the detection limit down to $0.001{ }^{\circ} \mathrm{C}$. The use of liquids with higher thermo-optic coefficients could increase the sensitivity. Transparent polymers are, as well, good candidates to implement compact and stable sensor heads with even higher sensitivity. 


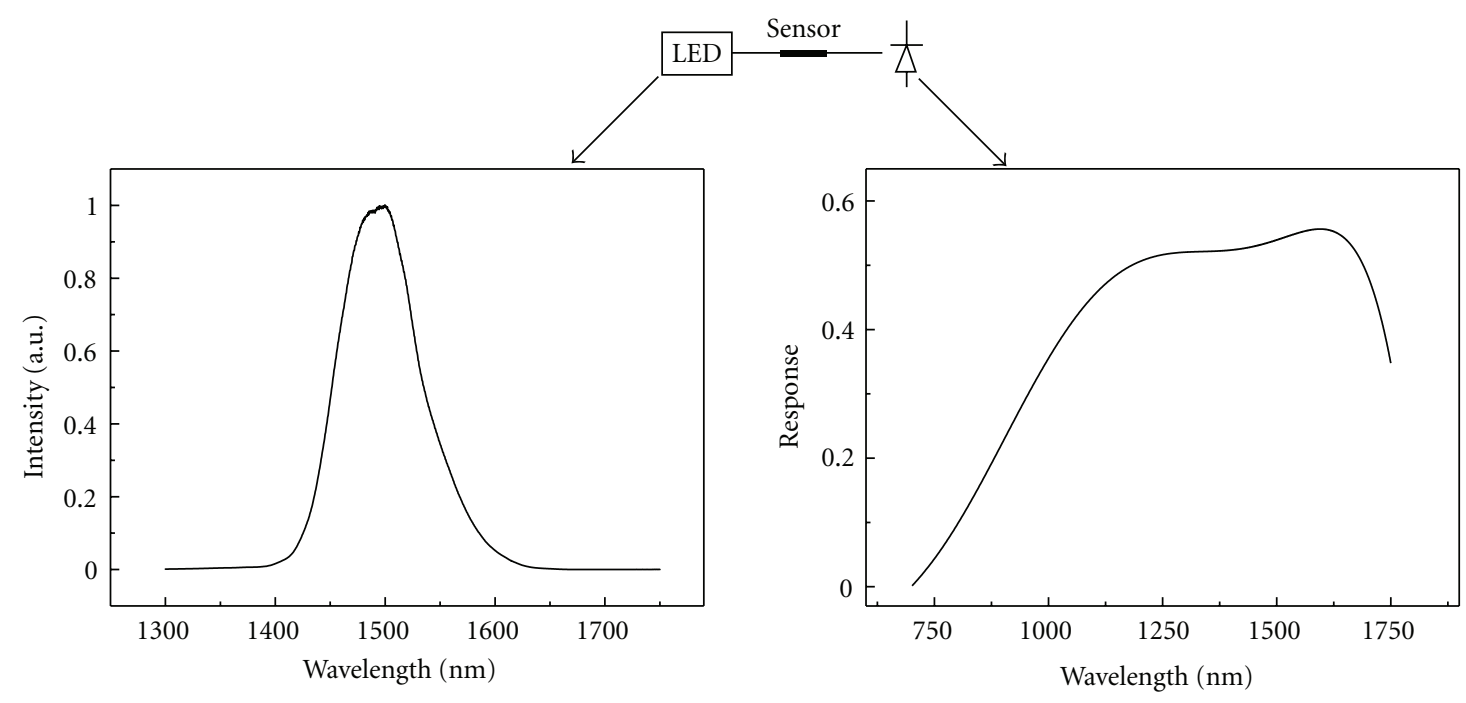

FIGURE 4: Schematic diagram of the sensor arrangement for amplitude interrogation. Optical spectrum of the LED and spectral response of the photodiode.

\section{Amplitude Interrogation}

One of the valuable characteristics of the previously reported temperature sensors is the wavelength codification of the sensor information. However, in some cases, one might be interested in using the simplest as possible interrogation technique. In such a case, our sensor heads permit the implementation of simple amplitude interrogation techniques. Figure 4 is a schematic diagram of the sensor arrangement that we have implemented to study the amplitude response of the sensors. A broadband source, either a light emitting diode (LED) or a halogen lamp (HL), was used to illuminate the sensor heads, and a photodiode was used to measure the transmitted power. Figure 4 includes the optical spectrum of the LED and the spectral response of the photodiode.

The amplitude response of the sensor is determined by the combination of the transmission characteristics of the sensor head as a function of temperature, the spectrum of the broadband light source, and the spectral response of the photodiode. Figure 5 gives two examples: (a) using a LED and (b) using an HL. In both cases, the sensor head was implemented using a section of fiber 2 and filling $31 \mathrm{~cm}$ with a liquid of RI $=1.47$. The smaller bandwidth of the LED makes the response to exhibit saturation, with a maximum slope of $0.127^{\circ} \mathrm{C}^{-1}$, while in the case of the HL the response is approximately linear $\left(\sim 0.022^{\circ} \mathrm{C}^{-1}\right)$. If we assume that the minimum relative amplitude change that we can detect is one thousandth, then the detection limit will be lower than $0.05^{\circ} \mathrm{C}$.

Figure 6 is a simulation of the response that should be obtained using the sensor reported in Figure 3 and measuring the transmission of a laser centred at $1510.25 \mathrm{~nm}$. The slope at $28.5^{\circ} \mathrm{C}$ is $0.353^{\circ} \mathrm{C}^{-1}$. Thus, if we assume that the transmittance can be measured with a resolution of one thousandth, then the detection limit would be $0.003^{\circ} \mathrm{C}$. The characteristics of this sensor configuration could be used for temperature stabilization. Changing the wavelength of the

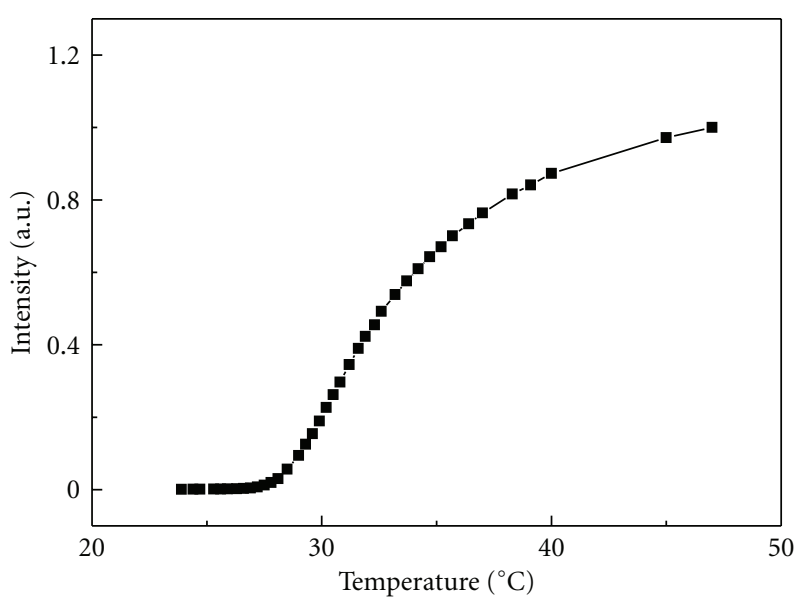

(a)

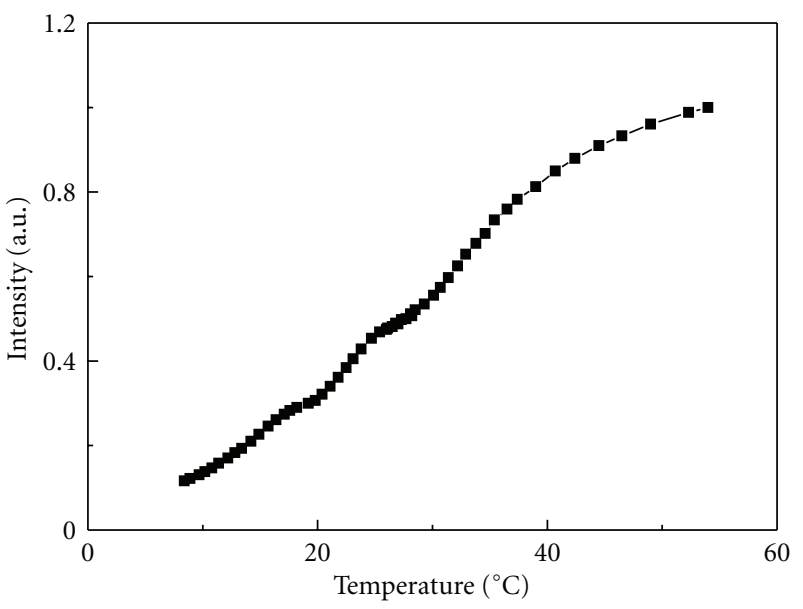

(b)

FIGURE 5: Calibration of the relative amplitude response of a sensor: (a) using a LED and (b) using a halogen lamp. 


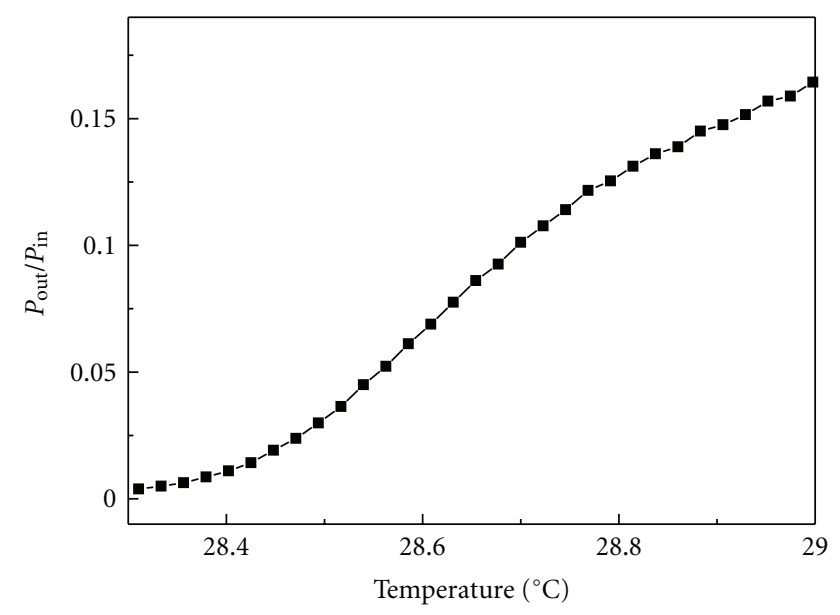

Figure 6: Transmittance of a sensor head at $1510.25 \mathrm{~nm}$ as a function of temperature.

laser, one could select the temperature of stabilization of the system.

\section{Conclusion}

Photonic crystal fibers open new opportunities by exploiting the unique properties that these fibers exhibit. Here we have exploited new possibilities based on the cutoff properties of the fundamental mode of Ge-doped photonic crystal fibers. Our experiments have been carried out using different Gedoped Y-shaped microstructured fibers, and sensor heads of different lengths and filled with different liquids have been implemented. The sensitivity is mainly determined by the thermo-optic coefficient of the liquids that fill the fibers. A sensitivity of $25 \mathrm{~nm} /{ }^{\circ} \mathrm{C}$ is reported, and a detection limit of about $0.001^{\circ} \mathrm{C}$ is estimated, when using the wavelengthencoded configuration. The sensor heads permit simple amplitude interrogation techniques to be implemented with high sensitivities and low detection limits.

\section{Acknowledgment}

This work has been financially supported by the Ministerio de Ciencia e Innovación of Spain (Project TEC2008-05490).

\section{References}

[1] B. Culshaw and J. Dakin, Optical Fiber Sensors: Systems and Applications, Artech House, 1989.

[2] K. T. V. Grattan and Z. Y. Zhang, Fiber Optic Fluorescence Thermometry, Chapman \& Hall, 1995.

[3] Y. J. Rao, "In-fibre Bragg grating sensors," Measurement Science and Technology, vol. 8, no. 4, pp. 355-375, 1997.

[4] L. Jin, W. Zhang, H. Zhang, et al., "An embedded FBG sensor for simultaneous measurement of stress and temperature," IEEE Photonics Technology Letters, vol. 18, no. 1, pp. 154-156, 2006.

[5] S. K. A. K. Bey, T. Sun, and K. T. V. Grattan, "Optimization of a long-period grating-based Mach-Zehnder interferometer for temperature measurement," Optics Communications, vol. 272, no. 1, pp. 15-21, 2007.

[6] F. Pang, W. Xiang, H. Guo, et al., "Special optical fiber for temperature sensing based on cladding-mode resonance," Optics Express, vol. 16, no. 17, pp. 12967-12972, 2008.

[7] H. Guo, F. Pang, X. Zeng, N. Chen, Z. Chen, and T. Wang, "Temperature sensor using an optical fiber coupler with a thin film," Applied Optics, vol. 47, no. 19, pp. 3530-3534, 2008.

[8] E. B. Li, X. L. Wang, and C. Zhang, "Fiber-optic temperature sensor based on interference of selective higher-order modes," Applied Physics Letters, vol. 89, Article ID 091119, 2006.

[9] E. Li and G. D. Peng, "Wavelength-encoded fiber-optic temperature sensor with ultra-high sensitivity," Optics Communications, vol. 281, no. 23, pp. 5768-5770, 2008.

[10] L. Rindorf and O. Bang, "Sensitivity of photonic crystal fiber grating sensors: biosensing, refractive index, strain, and temperature sensing," Journal of the Optical Society of America $B$, vol. 25, no. 3, pp. 310-324, 2008.

[11] H. Y. Choi, K. S. Pack, S. J. Park, U. C. Paek, B. H. Lee, and E. S. Choi, "Miniature fiber-optic high temperature sensor based on a hybrid structured Fabry-Perot interferometer," Optics Letters, vol. 33, no. 21, pp. 2455-2457, 2008.

[12] S. Torres-Peiró, A. Díez, J. L. Cruz, and M. V. Andrés, "Fundamental-mode cutoff in liquid-filled Y-shaped microstructured fibers with Ge-doped core," Optics Letters, vol. 33, no. 22, pp. 2578-2580, 2008.

[13] L. G. Coen, D. Marcuse, and W. L. Mammel, "Radiating leaky-mode losses in singlemode lightguides with depressedindex claddings," IEEE Transactions on Microwave Theory and Techniques, vol. 30, pp. 1455-1460, 1982. 

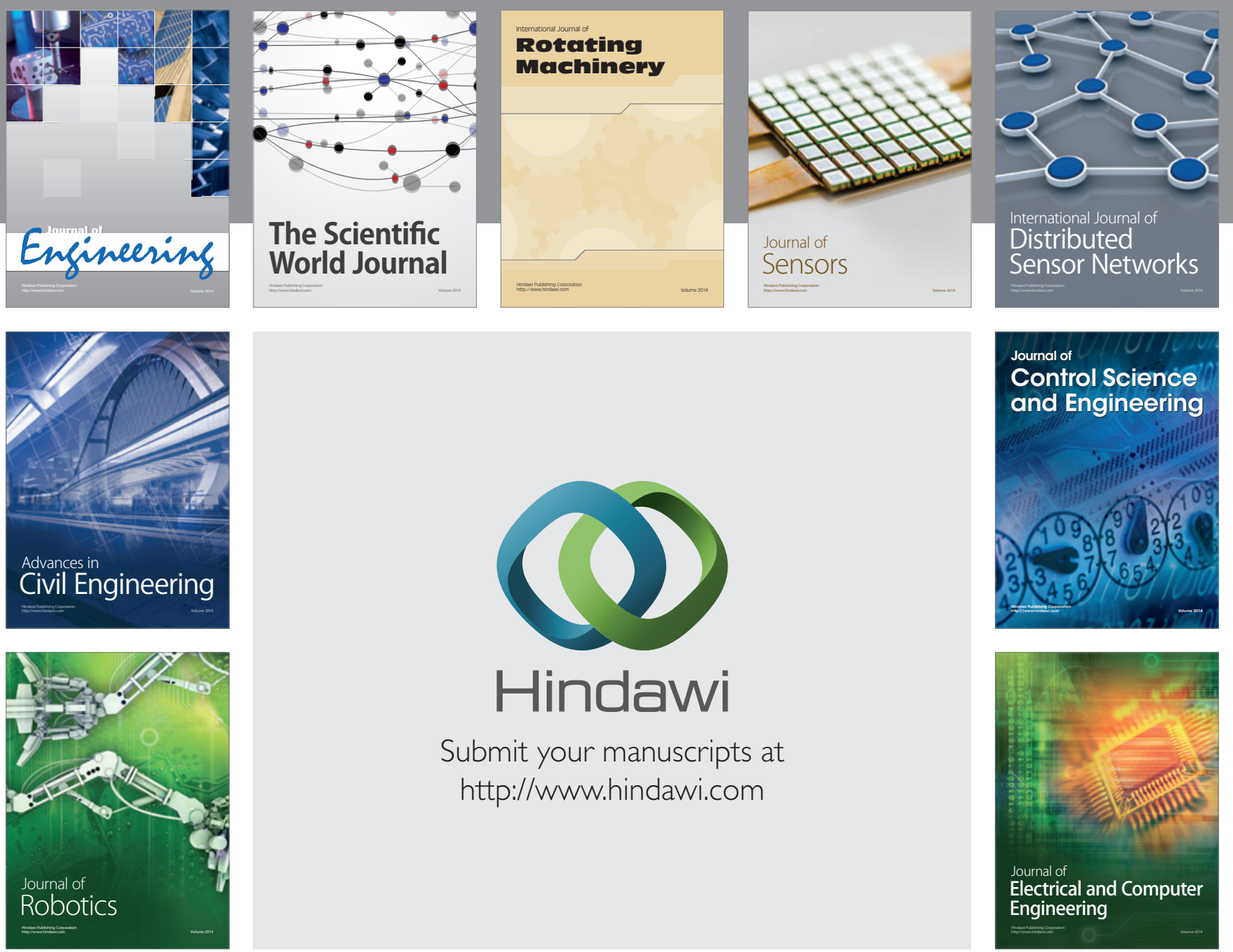

Submit your manuscripts at

http://www.hindawi.com
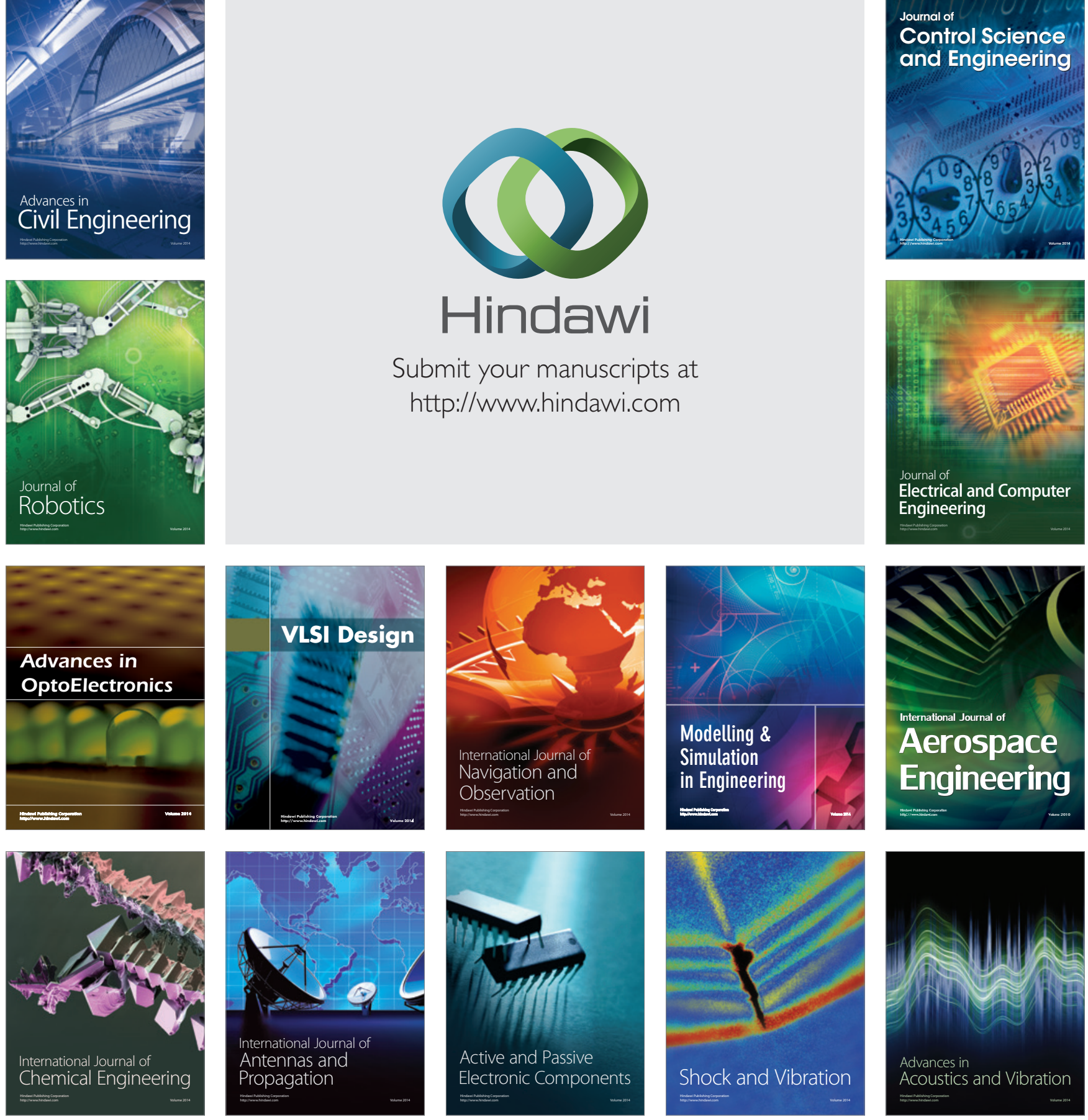